\title{
Analysis of quality characteristics in the multicriteria synthesis problem of the production schedule
}

\author{
Olga Chengar ${ }^{1, *}$, Elena Mashchenko ${ }^{1}$, Darya Zamorenova $^{2}$, and Andrey Phedosov $^{3}$ \\ ${ }^{1}$ Sevastopol State University, Department of Information Technologies and Computer Systems, 299053, University Str., Sevastopol, \\ Russian Federation \\ ${ }^{2}$ Sevastopol State University, Department of Instrument Systems and Automation of Technological Processes, 299053, University \\ Str., Sevastopol, Russian Federation \\ ${ }^{3}$ Federal state owned military educational institution of the Higher professional Education "the black sea higher navy order of the red \\ star academy by P.S.Nakhimov", 299028, Dybenko Str., Sevastopol, Russian Federation
}

\begin{abstract}
The article considers the possibility of applying the Pareto optimization method in combination with the ant algorithm for solving the task of scheduling the loading of process equipment in a flexible production system. Quality characteristics of the obtained production schedule of the machining section are analyzed depending on the choice of various combinations of optimization criteria. As a result of the experiments, carried out in the test case, it was found that with increasing number of criteria, the number of iterations required for a sufficiently accurate description of the Pareto boundary increases, which entails an increase in the algorithm running time, but the quality characteristics do not decrease significantly.
\end{abstract}

\section{Introduction}

There is currently considerable experience in addressing the challenges of building a discrete type production schedule, which is represented in the works of both domestic and foreign experts [1-5]. However, with the increase in the degree of automation of industrial enterprises, there is a growing need for increasingly powerful intelligent systems for short-term production planning, especially for in-house planning to compile a detailed schedule for loading technological equipment in a real production environment. Note that the use of known classical methods that explicitly or implicitly assume various simplifications in the formulation of the planning problem often does not yield acceptable results. Effective means for solving such problems is to use the methods of «Natural computing », which allow to receive sub-optimal solutions to problems of real work situations in a short time [1,3]. Ant algorithms (AA) are the prominent representative of «Natural computing " and they are based on the behavior of an ant colony as a population of potential solutions and adapted to solve combinatorial optimization problems, first of all, search for different paths on graphs $[2,4.6]$. Thus, it can be argued that AA have shown themselves to solve combinatorial optimization problems, one of which is the task of scheduling production equipment for flexible production systems (FPS).

Evaluating the effectiveness of the resulting production schedules can be made by one of the criteria:

- minimizing the violation as the deadlines for orders;

- maximizing the average load factor of flexible manufacturing modules (FMM);

- minimization of the cycle time manufacturing parts;
- minimizing the time changeovers;

- uniform loading equipment;

- minimization of not completed production;

- minimization of the number of using FMM.

However, it does not create the best in a real production environment, but rational plan of production. In connection with this approach is valid, providing the best using of not one efficiency criterion, and using the groups of criteria [7]. Priorities can change quickly based on current production needs. So, in practice, in a real production environment need to create a schedule of works, based simultaneously on a number of criteria, and thus the resulting timetable should be sub-optimal with respect to the selected criteria simultaneously. So the task of multi-criteria optimization becomes urgent in the production schedule, based on the method of ant colonies, the difficulty of working with a prospective nature of optimality conditions for a variety of criteria.

\section{Pareto optimization on the basis of the "direction" of ant algorithms with adaptive weights}

In $[7,8]$, it is proposed the Pareto method, which optimizes the production schedules based on the "directional" ant algorithm "to the account several factors affecting the production planning. To ensure a high efficiency of the production area it is proposed to form a schedule FPS download, which is the result of the decision multicriteria based on Pareto dominance concept.

When the multi-criteria optimization is searched more than one way of artificial populations of ants on the

\footnotetext{
* Corresponding author: OVChengar@sevsu.ru
} 
tops of the graph, and a variety of options for ways to each population, are optimal in the sense of Pareto. Therefore, the central issue is to form the target function. The analysis [9.10] showed the efficiency of the method of the weighted sum, wherein the weight is determined for each criterion, entering into the objective function. This approach is one of the most popular and natural development of classical optimization methods, where the "general" objective function is constructed from separate objective functions in the form of a weighted sum:

$$
F(x)=\sum_{i=1}^{k} w_{i} f_{i}(x),
$$

where weights are

$$
\mathrm{w}_{\mathrm{i}} \in[0,1] \text { and } \sum_{\mathrm{i}=1}^{\mathrm{k}} \mathrm{w}_{\mathrm{i}}=1
$$

The criteria were selected proceeding from the fact that optimization goals did not contradict each other, optimization was carried out in one direction (minimization or maximization), and also based on the requirements of the real production situation. Based on the above, three efficiency criteria were selected, the combinations of which are supposed to be experimentally investigated to find the effective solution of the optimal problem in the sense of Pareto:

- minimization of violation of deadlines for orders;

- minimization of the duration of the cycle of manufacturing parts;

- minimizing the time for equipment changes.

There are different opportunities and the various staging and tasks to optimize the production schedule for this search. In all productions, it is required among all possible ways of the graphic and analytic model to find a solution in which the resulting schedules will be the best.

There are combinations of possibility proposed performance criteria for further experimental studies:

I) minimize the violation of the deadlines for the manufacture of the order with the minimum duration of the cycle of manufacturing parts;

$$
\begin{gathered}
Z_{1}=\min f_{i}(x)=T f_{i}(x)-\operatorname{Tpl}_{i}(x),(i=1, \ldots, n) \\
Z_{2}=\min _{i}(x)=\sum_{i=1}^{n}\left(\sum_{j=1}^{m} \operatorname{To}_{i j}(x)+\sum_{j=1}^{m} \operatorname{Tnp}_{i j}(x)\right),(i=1, \ldots, n ; j=1, \ldots, m)
\end{gathered}
$$

II) minimize the violation of the deadlines for the manufacture of the order while minimizing the adjustment times of the equipment;

$$
\begin{gathered}
Z_{1}=\min f_{i}(x)=\operatorname{Tf}_{i}(x)-\operatorname{Tpl}_{i}(x),(i=1, \ldots, n) \\
Z_{2}=\min _{i}(x)=\sum_{i=1}^{n} \sum_{j=1}^{m} \operatorname{Tn}_{i j}(x),(i=1, \ldots, n ; j=1, \ldots, m)
\end{gathered}
$$

III) minimize the length of the manufacturing of parts while minimizing the adjustment times of equipment;

$$
\begin{aligned}
& Z_{1}=\min f_{i}(x)=\sum_{i=1}^{n}\left(\sum_{j=1}^{m} \operatorname{To}_{i j}(x)+\sum_{j=1}^{m} \operatorname{Tn} p_{i j}(x)\right),(i=1, \ldots, n ; j=1, \ldots, m) \\
& Z_{2}=\min _{i}(x)=\sum_{i=1}^{n} \sum_{j=1}^{m} \operatorname{Tn}_{i j}(x),(i=1, \ldots, n ; j=1, \ldots, m)
\end{aligned}
$$

Next in the series we will examine these options for multi-criteria optimization for load planning task $\mathrm{m}$ in FMM machining manufacturing site for production of $n$ batches of parts $\mathrm{x}$, simultaneously on two performance criteria proposed in $[7,8]$.

It was experimentally found that during the production program for a month, it is consisting of 3 parts in a total amount of about 200 pcs., The maximum number of iterations necessary to obtain a Pareto suboptimal solutions of $n_{t}=100$.

In Table 1, the experimentally obtained values of the parameters of the "directed" ant algorithm for the multicriterial optimization problem for the selected two targets Z1 and Z2 are shown simultaneously for the above selection of efficiency criteria.

The convergence of the algorithm depends largely on the following control parameters $[3,6]$ :

- the maximum number of iterations $\mathrm{n} t$;

- value of the initial concentration of pheromone $\tau 0$ at the transition artificial ants vertex graphic and analytic models corresponding to manufacturing operations;

- value of the initial concentration of pheromone in the transition $\tau 0$ "elite" artificial ants vertex graphic and

\begin{tabular}{|c|c|c|c|c|}
\hline \multicolumn{2}{|c|}{ Options $\quad$ Criteria } & I & II & III \\
\hline Denomination & Range of & Value & Value & Value \\
\hline $\begin{array}{l}\tau_{0}(\text { for all } \\
\text { ants })\end{array}$ & $(0 ; 1)$ & 0,090 & 0,150 & 0,095 \\
\hline $\begin{array}{l}\tau_{0} \text { (for "elite" } \\
\text { ants) }\end{array}$ & $(0 ; 1)$ & 0,380 & 0,310 & 0,425 \\
\hline$\alpha$ & $(0,3 ; 0,5)$ & 0,39 & 0,34 & 0,41 \\
\hline$\beta$ & $(0,5 ; 0,8)$ & 0,67 & 0,73 & 0,71 \\
\hline
\end{tabular}
analytic models corresponding storage operations;

- the value of the positive constant $\alpha$, which determines the effect of pheromone concentration;

- value of the positive constant $\beta$, which determines the effect of heuristic information.

Table 1. The control parameters of ant algorithm for finding Pareto-optimal solutions based on two criteria

Figure 1 is a graphical implementation of the results obtained by the first variant by selection criteria. For greater clarity, provided the task is displayed in the space of criteria in order of approximation of solutions to the Pareto-optimal.

The points are marked by the principle for all the points dominated the points obtained during the algorithm. Straight noted successive approximation line to the adaptive non-dominated solutions from the amount of artificial ant populations. The point of the conditional minimum, that is the solution of the problem under consideration, is the lower point of intersection of the admissible region with the adaptive line, built on a given number of iterations. 


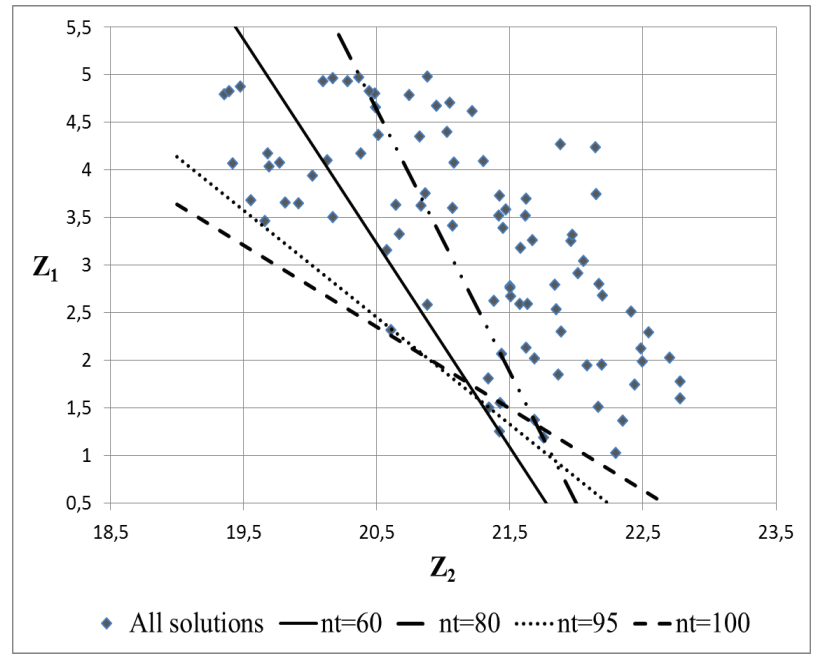

Fig.1. Pareto-optimization of the two-criteria problem: minimization of the violation of the deadlines for manufacturing the order with the minimum duration of the manufacturing cycle of parts.

Similar actions were also made for the second and the third variants of selection criteria and results are summarized in Table 2.

Table 2. Results of the solution of test tasks

\begin{tabular}{|c|c|c|c|}
\hline $\begin{array}{c}\text { Variant } \\
\text { of the } \\
\text { criterion } \\
\text { choice }\end{array}$ & $\begin{array}{c}\text { min duration } \\
\text { manufacturing } \\
\text { parts, h. }\end{array}$ & $\begin{array}{c}\text { min } \\
\text { time adjustment } \\
\text { of FMM, h. }\end{array}$ & $\begin{array}{c}\text { min } \\
\text { violation of } \\
\text { deadlines, h. }\end{array}$ \\
\hline I & 20,4 & - & 1,25 \\
\hline II & - & 5,04 & 1,45 \\
\hline III & 19,1 & 6,75 & - \\
\hline
\end{tabular}

The results of the solution of the proposed test tasks by the two-criteria "directional" ant algorithm make it possible to conclude that the modification of the method of ant colonies and the concept of Pareto dominance have been carried out-the points are located almost uniformly and over the whole set of criteria, the adaptive straight line moves successively to the unmodified points, thus approaching the Pareto-Optimal solution.

Similar studies were carried out for a problem with three criteria. According to their results, we can conclude that with increasing amounts of a growing number of criteria the population of artificial ants are increasing too, and it is needed for sufficiently accurate description of the Pareto frontier, which entails an increase in the operation time of the algorithm. In addition, the effectiveness of the solutions obtained by individual criteria is noticeably reduced, which is far from always justified in the real production environment.

\section{Analysis of the characteristics of the quality of production schedules}

For experimental studies and validation of the developed models for capacity planning process equipment as an object was selected organizational workflow process area of machining parts, such as bodies of rotation, with buildings in PJC "Azovobschemash" (Mariupol). This production site is designed for machining couplings, washers, disks, nipples of locks, rings with a diameter of up to $400 \mathrm{~mm}$ and a length of up to $500 \mathrm{~mm}(60-70$ thousand pieces / year with two shift operation), it has a medium-series production type. On this site, for each product an average of 3 to 5 operations, depending on its type, and up to 12 items can be processed. There is a block diagram of a portion of machining in Fig.2. It is composed of six cargo-lifting equipment, transport module and an automated warehouse.

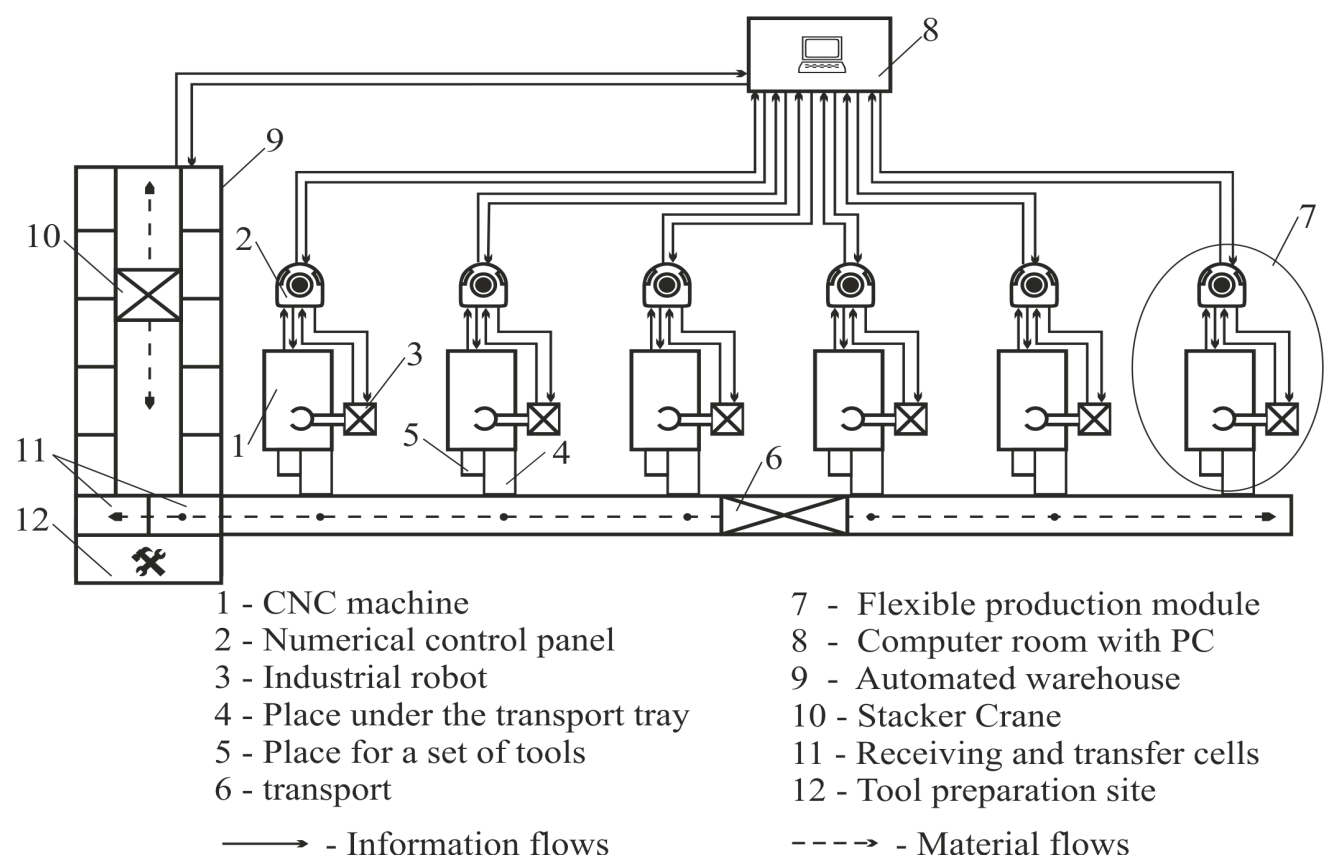

Fig.2. Structural diagram of the production section of machining parts such as bodies of rotation 
In the process of functioning of the FPS site machining with time, there is a need to change the nomenclature of manufactured products, production program, etc. Clarification of all transformations of the production site allows to ensure high efficiency of work in the changed operating conditions. With reference to the selected object of research, a series of computational experiments was carried out. To assess the adequacy of the model in production were selected information on the work performed in a few month $\mathrm{s}$ in order to compare with the results obtained in E experimentally on the basis of the developed model. At the same time estimated and camping by the following factors:

- observance of works and technological operations in the model of cause-effect relationships;

- obtaining planned indicators of the schedule (loading, downtime and equipment changeover, compliance with the deadlines).

Since the production site under consideration is built on the basis of CNC machines of the same technological purpose, the analysis confirmed the relative uniformity of loading and downtime of the FMM. Losses on readjustment are small enough and make up about 3.5\%, and downtime is about $25 \%$ of the total simulation time.

The main reasons for downtime are:

- absence of blanks (7\%);

- lack of tools $(5 \%)$;

- waiting for service by the transport module (10\%);

- equipment breakdown (2\%);

- other reasons $(1 \%)$.

The above mentioned data show that the automated transport can not cope with the performance of applications coming from FMM. Thus, the main possibility of "sealing" the schedule is to reduce the downtime of the process equipment for the first three reasons mentioned above. Also, analysis of indicators of the functioning of the automated transport it showed that its load factor is close to 1 , significantly exceeds the load lifting equipment, as The FPS is serviced by one transport, which moves the blanks and tools in two directions.

Therefore, the transport system has always been the bottleneck of the FPS and the issue of how to unload the transport is acute, thereby increasing the loading factor of the FMM.

With the application of the developed methods has been set up under the proposed model of FPS parameters described above. The evaluation of the results showed the observance of cause-effect relationships in all experiments and a significant improvement in the key parameters of the production schedule.

To analyze the production schedule obtained, the data on the performance of the production program, obtained after the implementation of the proposed model, are summarized in Table 3, which is built on the diagram (Fig.3), clearly showing the results of the experiment.

In the course of the experiment, multicriteria problems with the use of a "directed" ant algorithm with adaptive weights are also considered.
Table 3. Average values of the parameters of the production program before and after modeling

\begin{tabular}{|l|l|l|l|l|}
\cline { 2 - 5 } \multicolumn{1}{c|}{} & \multicolumn{2}{c|}{$\begin{array}{c}\text { Before } \\
\text { simulation }\end{array}$} & \multicolumn{2}{c|}{$\begin{array}{c}\text { After } \\
\text { simulation }\end{array}$} \\
\hline $\begin{array}{c}\text { Type of } \\
\text { equipment }\end{array}$ & FMM & Transport & FMM & Transport \\
$\begin{array}{l}\text { Average } \\
\text { value }\end{array}$ & 0,040 & 0,162 & 0,028 & 0,085 \\
\hline $\begin{array}{l}\text { The share of } \\
\text { adjustment }\end{array}$ & 0,283 & 0,030 & 0,186 & 0,010 \\
\hline $\begin{array}{l}\text { The share of } \\
\text { idle time }\end{array}$ & 0,690 & 1,000 & 0,801 & 0,940 \\
\hline $\begin{array}{l}\text { Coefficient of } \\
\text { loading }\end{array}$ & & & & \\
\hline
\end{tabular}

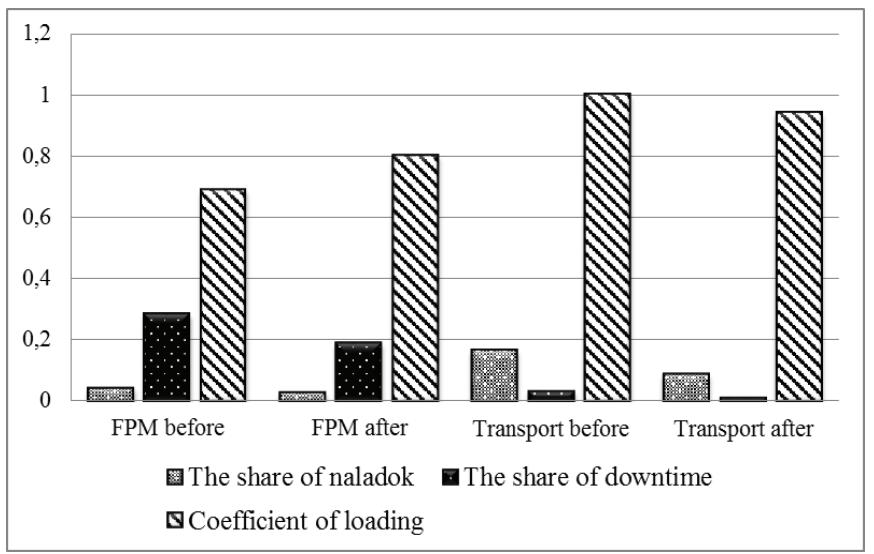

Fig.3. The diagram of the averaged values of parameters of loading, idle times and adjustments of the process equipment before and after modeling

The analysis of the results was carried out in comparison with the solutions obtained in solving the single-objective problem. The following options for choosing the optimality criteria were considered:

I. Maximizing the average load factor of process equipment

II. Minimization of the violation of the deadlines for the manufacture of an order with the minimum duration of the cycle of manufacturing parts

III. Minimization of the violation of the deadlines for the manufacture of the order, while minimizing the adjustment times of equipment

IV. Minimization of the violation of the deadlines for the manufacture of an order with the minimum duration of manufacturing of parts and the time of adjustment of equipment

Studies basic quality characteristics obtained by solving the optimization problem of the production schedule, have shown that the introduction of additional performance criteria, the task of choosing the optimal solution much more complicated, but the effectiveness of methods of an existing member is not reduced. So in Fig. 4 the graphs are showing the difference between the values of the basic obtained quality characteristics with one-criterion and multiobjective optimization. 


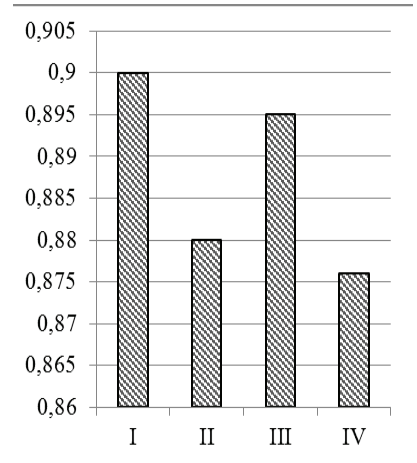

a) the average load factor $\mathrm{K} \mathrm{z}_{\mathrm{sr}}$,h.

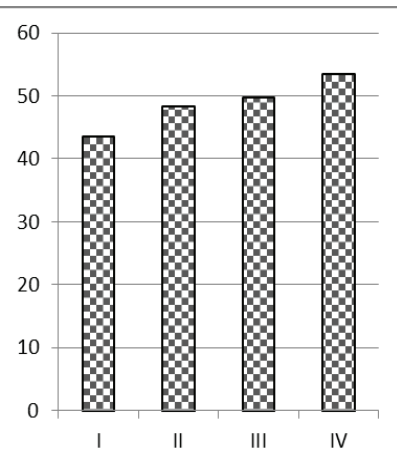

c) the equipment set-up time Tn,h.

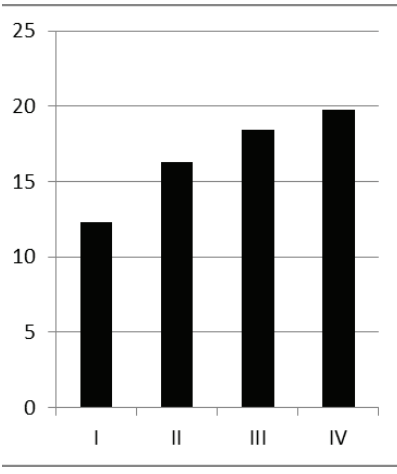

b) the time of the violation of deadlines $\mathrm{T}_{\mathrm{sr}}, \mathrm{h}$.

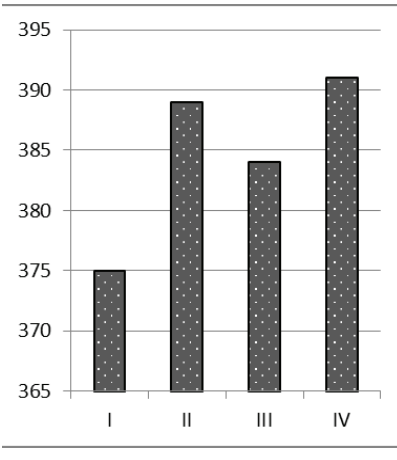

d) the duration of production cycle Tc,h.

Fig. 4. The values of the quality characteristics of the production schedule, depending on the choices of performance criteria

An experimental study of the Pareto optimization method for the production schedule of FPS based on a "directed" ant algorithm with adaptive weights allows us to assert that, according to various criteria of effectiveness, the discrepancy between the results of one-criterion and two-criteria problems is in the range of 1 to $3 \%$, and the one-criterion and three-criteria problems are within $6 \div 8,5 \%$, which is acceptable. Also made computer experiments have shown that the effectiveness of the directional ant algorithm increases with the dimension of the problem.

\section{References}

1. M. Dorigo Swarm Intelligence, Ant Algorithms and Ant Colony Optimization, Reader for CEU Summer University Course «Complex System». P. 1-34. (2001)

2. Y. Skobtsov, D. Speransky, Evolutionary computation (The National Open University "INTUIT", 2015)

3. O. Chengar E. Savkova, Journal of East Ukrainian National University 13 (167), P. 239-245 (2011)

4. O. Chengar, Journal of SFU. Technical Science, 4 (141), P. 185-193 (2013)

5. Y. Skobtsov, A. Sekirin, S. Zemlyanskaya, O. Chengar, V. Skobtsov, S.Potryasaev, Advances

in Intelligent Systems and Computing, vol.466, P.453-462 (2016)

6. O. Chengar, Bulletin of Kherson National Technical University, 1 (46), P. 212-217 (2013)

7. Y. Skobtsov, O. Chengar, V. Skobtsov, A.Pavlov. Advances in Intelligent Systems and Computing, vol.573, P.456-465 (2017)

8. O. Chengar, Problems of Information Technology. Bulletin of Kherson National Technical University, 1 (15), P. 211-216 (2014)

9. E. Zitzler, K. Deb, L. Thiele, Comparison of Multiobjective Evolutionary Algorithms, Vol. 8(2), P. 173-195 (2000)

10. K. Deb, Multi-objective optimization using evolutionary algorithms (UK: Wiley. 2001) 